\title{
Magnetic, Structural, and Optical Properties of Low Temperature ZnMnO Grown by Atomic Layer Epitaxy
}

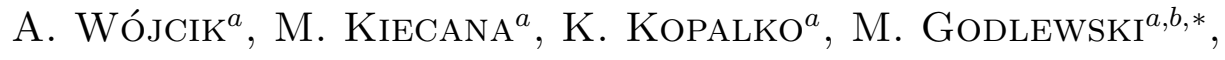
E. Guziewicz ${ }^{a}$, S. Yatsunenko ${ }^{a}$, E. Łusakowska ${ }^{a}$,

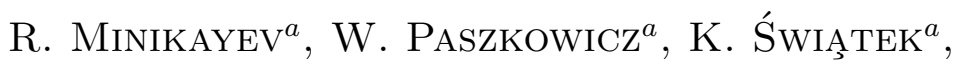
Z. Wilamowski ${ }^{a}$, M. SAWicki ${ }^{a}$ AND T. Dietr ${ }^{a}$

${ }^{a}$ Institute of Physics, Polish Academy of Sciences al. Lotników 32/46, 02-668 Warsaw, Poland

${ }^{b}$ Dept. Mathematics and Natural Sciences College of Science

Cardinal S. Wyszyński University, Warsaw, Poland

\begin{abstract}
Magnetic, structural, and optical properties of $\mathrm{ZnMnO}$ films grown with atomic layer epitaxy are discussed. Atomic layer epitaxy films were grown at low temperature using organic zinc and manganese precursors. From magnetometry and electron spin resonance investigations we conclude that lowering of a growth temperature significantly limits formation of Mn precipitates and inclusions of different foreign phases of manganese oxides to $\mathrm{ZnMnO}$ host.
\end{abstract}

PACS numbers: 68.55.Jk, 68.55.Ln, 68.55.Nq, 78.66.Hf, 81.15.Kk

\section{Introduction}

$\mathrm{ZnO}$, once doped with transition metal elements, is a promising material for applications in spintronics $[1,2]$. Theoretical estimations predict ferromagnetic (FM) phase in $p$-type $\mathrm{ZnMnO}$ (room temperature (RT) FM for $5 \%$ Mn fraction and $3.5 \times 10^{20} \mathrm{~cm}^{-3}$ hole concentration [1]) and in $n$-type $\mathrm{ZnCoO}$ [2]. The following experimental observations for $\mathrm{ZnMnO}$ (also for $\mathrm{ZnCoO}$ ) confirmed or rejected the presence of FM ordering, leading to a quite confusing situation. RT FM phase was reported by Sharma et al. [3], Lee et al. [4], Lim et al. [5], Theodoropoulou et al. [6], low temperature FM phase was reported by Jung et al. [7], spin glass

*corresponding author; e-mail: godlew@ifpan.edu.pl 
phase was reported by Fukumura et al. [8], whereas Tiwari et al. [9] and Kolesnik et al. [10, 11] and Kim et al. [12] did not find any FM ordering. Kolesnik and co-workers related FM phase reported by other groups to inclusions of foreign phases of Mn oxides.

Origin of inclusions of $\mathrm{Mn}$ oxides in $\mathrm{ZnMnO}$ was discussed by Sharma et al. [3]. From X-ray diffraction (XRD) they evidenced the presence of several manganese oxides in their $\mathrm{ZnMnO}$ samples, including $\mathrm{Zn}_{2} \mathrm{Mn}_{3} \mathrm{O}_{8}, \mathrm{ZnMn}_{3} \mathrm{O}_{7}, \mathrm{Mn}_{2} \mathrm{O}_{3}$, $\mathrm{Mn}_{3} \mathrm{O}_{4}$ (known to be either FM or ferri-magnetic), $\mathrm{Mn}_{5} \mathrm{O}_{8}$. In general, the oxide type and its quantity depend on a growth conditions. Most likely these oxides are preferably formed at increased growth temperatures of $\mathrm{ZnMnO}$. In particular, magnetic inclusions of $\mathrm{Mn}_{3} \mathrm{O}_{4}$ are expected to be dominant in samples prepared at high temperatures (heated to $1100^{\circ} \mathrm{C}[3]$ ). They however were not observed in samples of Kim et al. even though they sintered $\mathrm{ZnO}$ and $\mathrm{MnO}_{2}$ powders at $1100^{\circ} \mathrm{C}[12]$.

The inclusions of Mn oxides have strong impact on magnetic properties of $\mathrm{ZnMnO}$ films [3], as it is also demonstrated in the present work. In this work we discuss magnetic properties of LT ZnMnO films grown with atomic layer epitaxy (ALE) using organic zinc and Mn precursors. The successful growth of such films was reported by us recently [13]. We show that even at very low concentration inclusions of foreign phases result in magnetic ordering in the films studied.

\section{Growth method and samples characterization}

ALE, which is a version of chemical vapor deposition (CVD) technique, allows use of a wide range of reaction precursors, including very reactive ones, not suitable for CVD or metal-organic CVD applications and, most importantly, those with relatively low evaporation temperatures, i.e., LT growth $\left(T<500^{\circ} \mathrm{C}\right)$ is achievable using ALE.

The latter property of ALE is used in the present study, in which we selected organic reaction precursors to increase growth rate, to lower growth temperature, and also to increase Mn fraction in our films. When we used inorganic precursors, high quality $\mathrm{ZnO}$ films were obtained [14], but $\mathrm{Mn}$ fractions in $\mathrm{ZnMnO}$ were too low for spintronics purposes.

In the present study we used zinc acetate $\left(\mathrm{Zn}\left(\mathrm{CH}_{3} \mathrm{COO}\right)_{2}\right)$ as an organic zinc precursor and water vapor as oxygen precursor. $\mathrm{ZnO}$ films are obtained due to a double exchange chemical reaction: $\mathrm{Zn}\left(\mathrm{CH}_{3} \mathrm{COO}\right)_{2}+\mathrm{H}_{2} \mathrm{O} \rightarrow \mathrm{ZnO}+$ $2 \mathrm{CH}_{3} \mathrm{COOH}$ at a substrate temperature of $300 \div 360^{\circ} \mathrm{C}$ (so-called ALE window) and for precursors temperature of $230 \div 250^{\circ} \mathrm{C}$. The growth rate was exceptionally high as for ALE, reaching $0.5 \mathrm{~nm} /$ cycle, i.e., 1 monolayer per cycle was deposited. Nitrogen gas as a transport and purging gas was used. All samples discussed in the present work were grown on a sapphire substrate. Two types of organic Mn precursors - $\mathrm{Mn}(\text { thd })_{3}\left(\right.$ at $\left.160^{\circ} \mathrm{C}\right)$ and $\mathrm{Mn}(\text { acac })_{3}\left(\right.$ at $\left.160 \div 180^{\circ} \mathrm{C}\right)$ - were used for the LT growth of $\mathrm{ZnMnO}$. 
XRD investigations were performed for all samples studied and discussed in the present work. These investigations are discussed in more details elsewhere [15]. XRD showed change of a preferential growth orientation for LT ZnO films grown using zinc acetate. $\mathrm{ZnO}$ films grown by us using inorganic zinc precursor $\left(\mathrm{ZnCl}_{2}\right)$ had $c$ axis perpendicular to a film surface. LT ZnO films as a privileged orientation had $c$ axis parallel to the substrate.

XRD also showed that Mn doping changes a volume of a crystal cell. Values of $c$ (increases) and $a$ (decreases) axis parameters change upon Mn doping. This formally should allow us to estimate Mn fractions in our films [8, 9, 12]. We found however that the dependences given in Refs. [8, 9, and 12] give surprisingly large errors in estimations of $\mathrm{Mn}$ fractions. For example, for one of $\mathrm{ZnMnO}$ films grown with $\mathrm{Mn}(\text { thd })_{3}$ precursor we got $5 \%$ Mn fraction using the dependence of $c$ vs. Mn concentration given in [8], about $20 \%$ using the analogous dependence given in [9], and well below $1 \%$ based on the similar dependence given in Ref. [12]. Such large spread of estimations of Mn fractions may likely be the consequence of large differences in amounts of inclusions of Mn foreign phases in the samples studied in Refs. [8, 9, and 12].

This is why we used several complementary experimental methods to estimate $\mathrm{Mn}$ fractions in our films. Mn fractions in our $\mathrm{ZnMnO}$ films were evaluated using XRD, optical absorption investigations (from shift of fundamental absorption band), secondary ion mass spectroscopy (SIMS) and electron probe micro-analysis (EPMA). Estimations from magnetization measurements (from SQUID magnetometry) are discussed below. XRD and SQUID gave similar Mn fractions (typically of about 5\%), EPMA underestimated Mn fractions, whereas SIMS investigations overestimated $\mathrm{Mn}$ fractions by nearly a factor of two.

Our recent investigations with atomic force microscopy (AFM) indicated that crystallinity of ALE ZnO films strongly depends on a lattice mismatch between $\mathrm{ZnO}$ and a substrate [13-15]. We obtained monocrystalline $\mathrm{ZnO}$ films on $\mathrm{GaN} / \mathrm{Al}_{2} \mathrm{O}_{3}$ and polycrystalline films on $\mathrm{Al}_{2} \mathrm{O}_{3}$, Si and lime glass substrates [13-15]. The present AFM investigations indicate that LT growth conditions and LT doping with Mn using organic precursors reduce crystallinity and surface topography of ALE films. All LT ZnMnO films studied in the present work were polycrystalline.

Good quality of thin films is usually confirmed by observation of band edge light emission. Well-resolved excitonic spectra were observed in either photoluminescence (PL) or cathodoluminescence (CL) of our LT $\mathrm{ZnO}$ films prior to the LT Mn doping [14]. These spectra, observed up to the room temperature, and also a defect related red-green PL show high in-plane homogeneity, as we concluded from the CL investigations not discussed here. Excitonic emission becomes very weak after Mn doping. We do not observe any Mn related intra-shell emission in a visible part of the spectrum, even for the films containing a few percent of $\mathrm{Mn}$ ions. 


\section{Magnetic properties of $\mathrm{ZnMnO}$ films}

We performed magnetic resonance investigations of two types of LT ZnMnO films discussed above. These investigations, performed using X-band electron spin resonance (ESR) (see Figs. 1 and 2a, b), confirmed Mn substitution to both types of our LT $\mathrm{ZnO}$ films. The ESR spectra observed for $\mathrm{Mn}(\mathrm{acac})_{3}$ and $\mathrm{Mn}(\mathrm{thd})_{3}$ were different. Whereas the typical paramagnetic response was observed in ESR of films doped with $\mathrm{Mn}(\mathrm{acac})_{3}$ (Fig. 2a), the response observed for the films doped with $\mathrm{Mn}(\text { thd })_{3}$ is more complicated (Fig. 1 and $2 \mathrm{~b}$ ). LT part of the latter ESR spectrum is dominated by a paramagnetic signal of "isolated" $\mathrm{Mn}^{2+}$ ions (see Fig. 1). This signal shows a weakly resolved 6 lines hyperfine structure of $\mathrm{Mn}^{2+}$. We assume that the signal comes from a small amount of $\mathrm{Mn}^{2+}$ ions accumulated at grain boundaries. Then, at slightly increased temperature, the ESR spectrum is dominated by a broad, featureless signal (Fig. 1). Its temperature dependence (Fig. 2b) suggests a super-paramagnetic-like response. This spectrum dominates up to about $200 \mathrm{~K}$ and is due to a majority of $\mathrm{Mn}^{2+}$ ions in our $\mathrm{ZnMnO}$ films. Then, for temperature above $200 \mathrm{~K}$, the ESR signal is replaced by another one

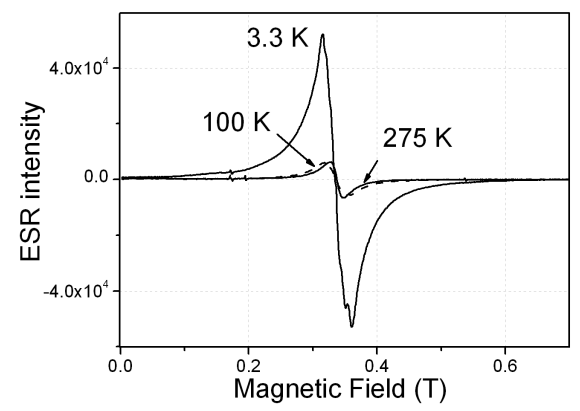

Fig. 1. The ESR spectra of $\mathrm{ZnMnO}$ grown with $\mathrm{Mn}(\text { thd })_{3}$ measured at 3.3, 100, and $275 \mathrm{~K}$.
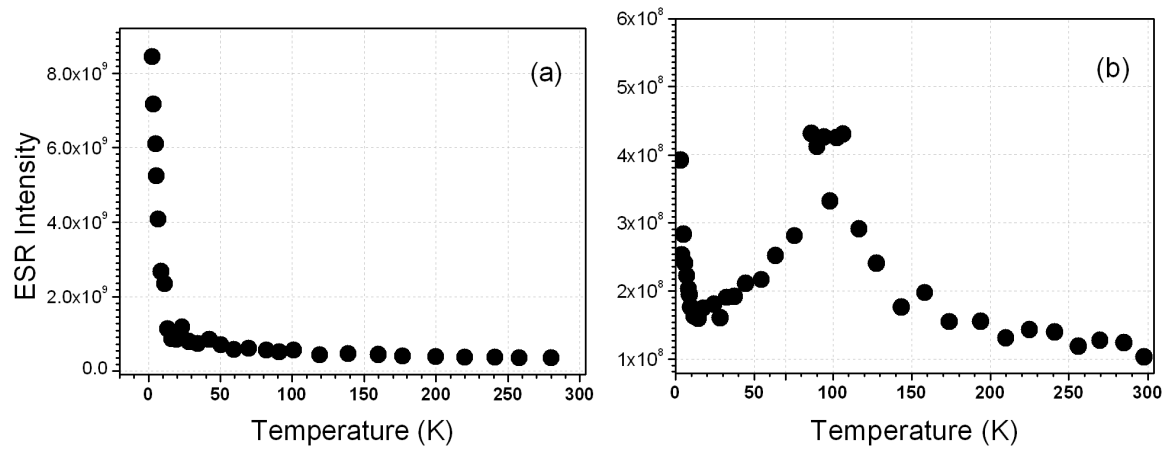

Fig. 2. Temperature dependences of intensity of $\mathrm{Mn}^{2+}$ ESR signals measured for two types of $\mathrm{ZnMnO}$ films: (a) for films grown with $\mathrm{Mn}(\mathrm{acac})_{3}$ and (b) for films grown with $\mathrm{Mn}(\text { thd })_{3}$ as $\mathrm{Mn}$ precursors. 
also in the form of a broad and featureless signal (Fig. 1). From the temperature dependence of the latter ESR signal we claim a weak contribution of FM phase, observed for temperatures above $200 \mathrm{~K}$ (Fig. 2b). We relate this signal to sparse inclusions of foreign phases of manganese oxides in our films.

Further information on magnetic properties of our films is drawn from magnetization investigations performed using a home made SQUID magnetometer. The magnetization curves were collected under both field cooling (FC, 1000 Oe) and zero field cooling (ZFC) conditions.

Assuming that foreign phases are missing in our films, featureless magnetization curves should be observed, closely matching the Curie law. Figures $3 \mathrm{a}, \mathrm{b}$ show however that the temperature dependences of magnetization measured for both types of our $\mathrm{ZnMnO}$ films deviate from the Curie law. Whereas high temperature parts of magnetization curves follow the Curie law, distinct deviations are observed at low temperatures. These deviations indicate contribution of additional magnetic moments to a measured sample magnetization and will be related later on to inclusions of Mn oxides. We will further show that amount of Mn ions in such inclusions is very low in our LT ZnMnO films.
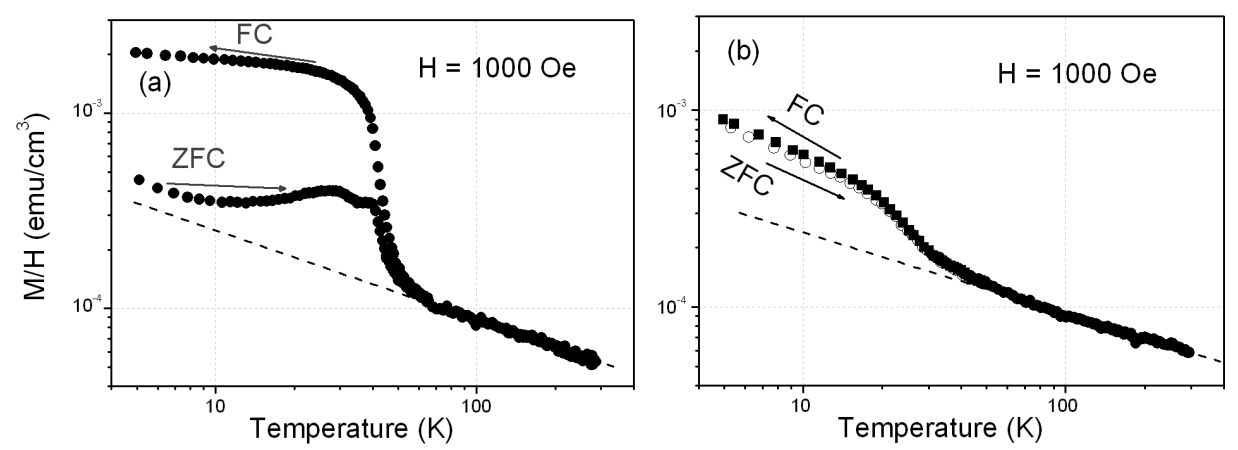

Fig. 3. Double logarithmic curves of magnetization versus temperature measured with and without (zero field cooling (ZFC)) magnetic field cooling (FC) for two types of ZnMnO films: (a) for films grown with $\mathrm{Mn}(\mathrm{acac})_{3}$ and (b) for films grown with $\mathrm{Mn}(\text { thd })_{3}$ as Mn precursors.

From high temperature parts of magnetization curves (see Figs. 3a, b) we estimate Mn composition in our LT ZnMnO films. Here (after introducing corrections for a sapphire substrate) we utilized the known relation between samples susceptibility and the Curie-Weiss temperature $(\Theta(x))$ and concentration $(x)$ of magnetic ions (from the Curie-Weiss law)

$$
\chi=C /(T-\Theta),
$$

where both the Curie-Weiss temperature, $\Theta$, and Curie constant $C$ depend on $\mathrm{Mn}^{2+}$ fraction in our sample 


$$
C=N\left(g_{\text {eff }} \mu_{\mathrm{B}}\right)^{2} S(S+1) / 3 k,
$$

where $S-$ spin of $\mathrm{Mn}^{2+}$ ions, $N$ - number of cations per unit volume, $g_{\mathrm{eff}}-$ effective Landé factor of $\mathrm{Mn}^{2+}$ ions, $\mu_{\mathrm{B}}$ - Bohr magneton, $k$ - Boltzmann factor.

We performed such estimation of Mn fraction for two selected LT ZnMnO films grown using identical ALE procedures but either $\mathrm{Mn}(\mathrm{acac})_{3}$ or $\mathrm{Mn}(\mathrm{thd})_{3}$ as the Mn precursors. From the estimations we obtained $8-9 \% \mathrm{Mn}$ fraction for samples doped using $\mathrm{Mn}(\mathrm{acac})_{3}$ and $12 \%$ for those grown with $\mathrm{Mn}(\mathrm{thd})_{3}$. We take these numbers as rather the upper limits for Mn contents in our films, since the $1 / C$ gets underestimated if even a small fraction of FM coupled phase is present, which in fact we observed in our investigations. If we correct our data for small amounts of Mn inclusions, concentration of substituting Mn ions in our films is reduced to $2-3 \%$ for samples doped using $\mathrm{Mn}(\mathrm{acac})_{3}$ and $6 \%$ for those grown with $\mathrm{Mn}(\mathrm{thd})_{3}$.

We did the latter correction since LT parts of magnetization curves, and also a large value of the remanence obtained for $\mathrm{Mn}(\mathrm{acac})_{3}$ samples, indicate the presence of foreign phase inclusions in our samples, which we relate to $\mathrm{Mn}_{3} \mathrm{O}_{4}$. From the LT part of magnetization curve we estimate concentration of these inclusions to be very low - no more than one $\mathrm{Mn}$ ion in $\mathrm{Mn}_{3} \mathrm{O}_{4}$ per $10^{4}$ substituting $\mathrm{Mn}$ ions. Remarkably, despite such a low concentration, the LT magnetic response is fully dominated by $\mathrm{Mn}_{3} \mathrm{O}_{4}$. It indicates how carefully we should draw conclusions on magnetic ordering in studied $\mathrm{ZnMnO}$ films.

It is interesting to underline that due to a low concentration of $\mathrm{Mn}_{3} \mathrm{O}_{4}$ these inclusions were not detected in our ESR measurements for films grown with $\mathrm{Mn}(\mathrm{acac})_{3}$. Interestingly, the ESR indicates the presence of a low concentration of other FM inclusions (their response dominates in ESR at high temperature) in the samples grown by us with $\mathrm{Mn}(\text { thd })_{3}$, not resolved in SQUID measurements. This indicates necessity of complementary SQUID and ESR investigations to conclude more reliable on magnetic ordering in a given sample.

\section{Conclusions}

Concluding, we report successful $\mathrm{Mn}$ doping of $\mathrm{ZnO}$, which is achieved in LT ALE growth processes. Using $\mathrm{Mn}(\mathrm{thd})_{3}$ we obtained homogeneous films of $\mathrm{Zn}_{1-x} \mathrm{Mn}_{x} \mathrm{O}$ with a dominant super-paramagnetic magnetic behavior, while films doped with $\mathrm{Mn}(\mathrm{acac})_{3}$ show LT ferromagnetic inclusions, which we attribute to the presence of foreign $\mathrm{Mn}_{3} \mathrm{O}_{4}$ phases. Even though their concentration is very low (for example not seen in XRD), they dominate low temperature magnetic properties of our films studied with SQUID. We underline also that SQUID and ESR investigations are complementary for estimation of magnetic ordering in the films. Whereas SQUID is very sensitive to inclusions, ESR detects contributions of dominant substituting Mn ions. Only if both techniques give the same information we can conclude on magnetic ordering in a given sample. 


\section{Acknowledgments}

This work was partly supported by grant no. PBZ-KBN-044/P03/2001 of the State Committee for Scientific Research. The ALE reactor was bought using SEZAM grant of Foundation for Polish Science.

\section{References}

[1] T. Dietl, H. Ohno, F. Matsukura, Science 287, 1019 (2000).

[2] K. Sato, H. Katayama-Yoshida, Jpn. J. Appl. Phys. 39, L555 (2000).

[3] P. Sharma, A. Gupta, F.J. Owens, A. Inoue, K.V. Rao, J. Magn. Magn. Mater. 282, 115 (2004).

[4] S. Lee, D.S. Kim, D.Y. Kim, Y.D. Woo, T.W. Kim, J. Korean Phys. Soc. 45, S787 (2004).

[5] S.W. Lim, M.C. Jeong, M.H. Ham, J.M. Myoung, Jpn. J. Appl. Phys. 43, L280 (2004).

[6] N.A. Theodoropoulou, A.F. Hebard, D.P. Norton, J.D. Budai, L.A. Boatner, J.S. Lee, Z.G. Khim, Y.D. Park, M.E. Overberg, S.J. Pearton, R.G. Wilson, Solid-State Electron. 47, 2231 (2003).

[7] S.W. Jung, S.-J. An, Gyu-Chul Yi, C.U. Jung, Sung-Ik Lee, S. Cho, Appl. Phys. Lett. 80, 4561 (2002).

[8] T. Fukumura, Z. Jin, A. Ohmoto, H. Koinuma, M. Kawasaki, Appl. Phys. Lett. 78, 958 (2001).

[9] A. Tiwari, C. Jin, A. Kvit, D. Kumar, J.F. Muth, J. Narayan, Solid State Commun. 121, 371 (2002).

[10] S. Kolesnik, B. Dabrowski, J. Mais, J. Appl. Phys. 95, 2582 (2004).

[11] S. Kolesnik, B. Dabrowski, J. Mais, Phys. Status Solidi C 1, 900 (2004).

[12] S.S. Kim, J.H. Moon, B.-T. Lee, O.S. Song, J. Appl. Phys. 95, 454 (2004).

[13] A. Wójcik, K. Kopalko, M. Godlewski, E. Łusakowska, W. Paszkowicz, K. Dybko, J. Domagała, A. Szczerbakow, E. Kamińska, Acta Phys. Pol. A 105, 667 (2004).

[14] K. Kopalko, M. Godlewski, J.Z. Domagala, E. Lusakowska, R. Minikayev, W. Paszkowicz, A. Szczerbakow, Chem. Mater. 16, 1447 (2004).

[15] A. Wójcik, K. Kopalko, M. Godlewski, E. Łusakowska, E. Guziewicz, R. Minikayev, W. Paszkowicz, K. Świạtek, M. Klepka, R. Jakieła, M. Kiecana, M. Sawicki, K. Dybko, M.R. Phillips, to be published. 